\title{
New Approach for Assembling Dissimilar Materials: Laser Technology
}

\author{
Anne Henrottin, Jérôme Patars, and Jose Antonio Ramos-de-Campos \\ Lasea, Laser Engineering Applications, Rue des Chasseurs Ardennais n¹0, B-4031, Belgium \\ E-mail: ahenrottin@lasea.com
}

\begin{abstract}
The research of new assembly technologies for dissimilar materials is growing up continuously mainly for avoiding the adding of chemical adhesive, assembly elements or additional materials, and gives the possibility to join complex geometries structures while maintaining their integrity. The present study proposes a new approach for developing high resistance hybrid joining between metal and polymer. This complete non-contact laser process allows to strongly join up to the breaking of the polymer part without affecting the joining area. The research has been focused on substrates having complex properties such as high reflectivity, thin thickness or transparent. Different laser sources with various pulse durations have been studied in order to evaluate their efficiency for a hybrid joining.
\end{abstract}

DOI: $10.2961 /$ jlmn.2018.03.0026

Keywords: laser micro-machining; hybrid joining; reflective metal; thin metallic substrates; polymer; lightweight applications

\section{Introduction and Motivation}

Nowadays, different laser technologies are used to weld metal with polymer. High powerful lasers, typically in the $\mathrm{kW}$ range, CW or long pulsed, are commonly used for the global process, structuration and joining $[1,3,4]$, in their research. This technology is well adapted for automotive industry for improving the lightweight constructions composed by opaque polymer at the laser wavelength inducing the joining by conduction, i.e. by heating directly the metal plate. Different approaches have already tested. Actually, Kah P., et al [2], worked on chemical bonding between dissimilar materials. For creating structures inside the metal plate, stamping process, sandblasting [3] and $\mu$ s-pulsed lasers [4] have also been explored. Amend, et al. [5] proposed another approach combining monochromatic laser radiation and additional polychromatic radiation for the joining process. The structuration process was carried out with short pulsed laser. This approach has shown the possibility to weld aluminum with transparent polycarbonate [6] but without reaching the rupture of the polymer while keeping intact the joining with the metal.

The present study proposes a new methodology for structuring high reflective, and with high thermal conductivity metallic plates and thin metallic foils for hybrid joining based on short and ultra-short pulsed lasers. Moreover, this new methodology allows to combine metal and polymer for applications having some technical limitations in terms of temperature reached during the joining phase or chemical modification (for example oxidation) of the metallic foil. Indeed, in the medical sector, Mian A., et al. [7], have shown the interest to combine, by laser, a thin metal sheet with polymer in the medical implant application. On one side, the encapsulation materials for permanent active medical implants seems to be more performant and to en- sure total air tightness when polymer is used. On another side, the laser process is a performant substitute technology for preventing damage effects due to traditional technologies such as hot-plate bonding, friction bonding or electromagnetic welding, as presented by N. Amanat [8]. The difficulty in the joining between completely different materials is the adhesion between them. In order to be independent to this feature, the present study is based on a two steps process: the first for ensuring the setting up of microstructuration playing the role of a clamping tool and the second one for heating progressively the polymer in order to melt it. The influence of the laser source in the hybrid joining process is studied in this research.

\section{Experimental}

\subsection{Material}

Four metal plates have been studied: titanium alloy (Ti90Al6V4, thickness $\mathrm{t}=100 \mu \mathrm{m}$ ) commonly used in implants, copper-O.F.H.C (thickness $\mathrm{t}=200 \mu \mathrm{m}$ ), stainless steel $(15-7 \mathrm{PH}$, thickness $\mathrm{t}=250 \mu \mathrm{m}$ and $\mathrm{t}=800 \mu \mathrm{m})$ and aluminum (EN AW-1200A, thickness $t=1 \mathrm{~mm}$, EN AW6063 and EN AW-5182, thickness $t=2 \mathrm{~mm}$ ). Concerning the polymer part, following the metal, the polymer to use can vary depending on the application. Indeed, for electronics applications, stainless steel, aluminum and copper will be associated to polycarbonate (various thicknesses will be tested; $\mathrm{t}=500 \mu \mathrm{m}, 1 \mathrm{~mm}$ and $2 \mathrm{~mm}$ ). For medical applications, alloy of titanium will be joined with Poly-L-lactic acid (biopolymer, thickness $\mathrm{t}=50 \mu \mathrm{m}$ ).

\subsection{Laser machines}

Fig. 1 shows Lasea's machine used for producing hybrid joining between various couples of metals and polymers. 


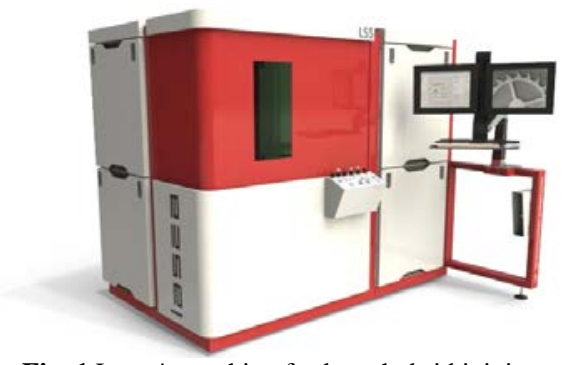

Fig. 1 Lasea's machine for laser hybrid joining.

The machine can have short and ultra-short pulsed lasers for the micro-structuration and CW lasers for the joining. Composed of linear and rotational axes, the machine can process complex geometries. In the study, three laser sources have been compared for the micro-structuration process: nanosecond pulsed laser $\left(\lambda=1064 \mathrm{~nm}, \mathrm{P}_{\max }=20 \mathrm{~W}\right.$, $\left.\mathrm{d}_{\mathrm{L}}=7.5 \mathrm{~mm}, \mathrm{t}=100 \mathrm{~ns}\right)$, picosecond laser $(\lambda=1064 \mathrm{~nm}$, $\left.\mathrm{P}_{\max }=30 \mathrm{~W}, \mathrm{~d}_{\mathrm{L}}=9 \mathrm{~mm}, \mathrm{t}=150 \mathrm{ps}\right)$ and femtosecond laser $(\lambda=$ $1030 \mathrm{~nm}, \mathrm{P}_{\max }=20 \mathrm{~W}, \mathrm{~d}_{\mathrm{L}}=2.5 \mathrm{~mm}, \mathrm{t}=300 \mathrm{fs}$ ). A CW diode laser $\left(\lambda=980 \mathrm{~nm}, \mathrm{P}_{\max }=200 \mathrm{~W}, \mathrm{~d}_{\mathrm{L}}=5 \mathrm{~mm}\right)$ has been used for the welding process. Complex vision system is also integrated inside the machine for ensuring exact positioning of the sample and quality control (Fig. 2).

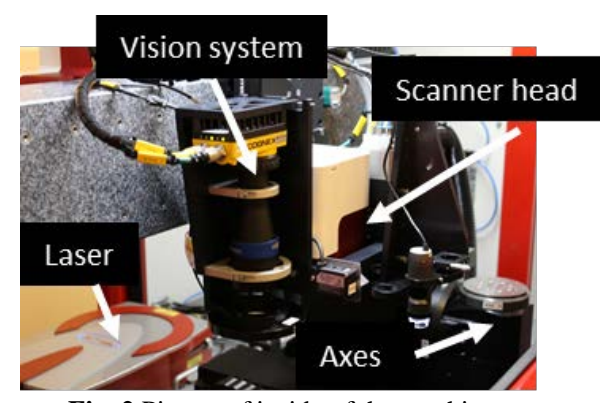

Fig. 2 Picture of inside of the machine.

\subsection{Laser micro-structuration process}

The geometry developed is based on lines having various orientation forming a specific pattern. After many experimental validations for selecting the most appropriate design, the chosen one consists in concentric lozenges. This design combines the effect of grooves with different orientations improving the clamping aspect and the achievement of a suitable ratio between the microstructure surface and the global joining area. Fig. 3 illustrates the geometrical parameters studied during the research. The dimensions of each design are a function of the groove width ' $W$ ' which depends on the laser source. The distance between concentric lozenges, named ' $h_{p}$ ', takes different values proportional to the line width. Furthermore, other parameters have been studied such as the distance between lozenges as their size as well.

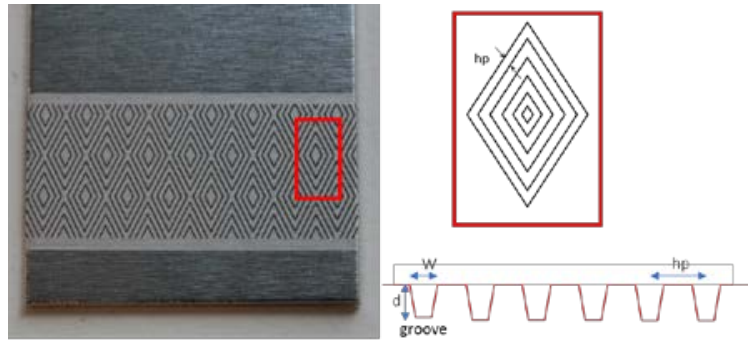

Fig. 3 Schematic representation of lozenge design.

The global micro-structure density (GMD) on the joining area has been defined for comparing the optimized results. Its mathematical expression is GMD $=\mathrm{W} / \mathrm{h}_{\mathrm{p}}$. In other terms, the GMD is a quantity representing a ratio between the surface treated by laser and the non-affected surface. It is important to note that the non-treated surface plays a role in the hybrid joining process, because it induces the transfer of heat between the metal and the polymer. A compromise between the number of grooves, ensuring the clamping, and the non-affected surface is the key of a good joining which are characterized by the rupture of the polymer keeping intact the joining area.

In the study, the joining surface treated by laser has been fixed at $2.5 \mathrm{~cm}^{2}$ on sample having $25 \mathrm{~mm}$ width and $80 \mathrm{~mm}$ length.

Fig. 4 presents SEM images for an example of microstructuration performed by laser. Depending on the laser sources and metal sheet, the laser parameters have to be adapted for reaching the rupture of the polymer without detaching the join.

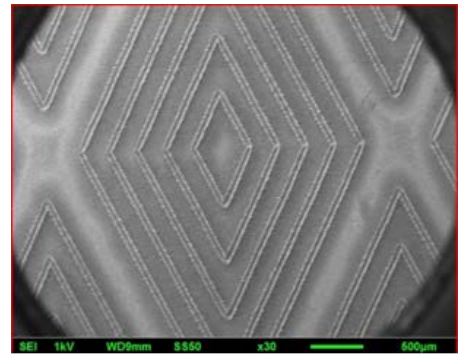

Fig. 4 SEM images of lozenge design.

The pulse duration of the laser source influences the quality of the grooves. Fig. 5 presents SEM images of grooves performed with (a) femtosecond laser and (b) nanosecond laser. Due to its longer pulse duration, the nanosecond laser induces more heat affected zones.
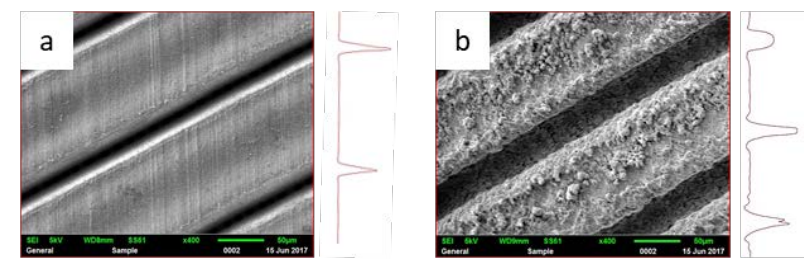

Fig. 5 SEM images of micro-structurations performed with (a) femtosecond laser and (b) nanosecond laser.

The heat created during the laser structuration process with nanosecond laser produces indirect effects as bending of the thin metal TiAl6V4 alloy sheet or oxidation on copper surface (Fig. 6). Depending on the application, these effects must be avoided for keeping the integrity of the 
surface. Whereas, using femtosecond laser allows to achieve clean surfaces.

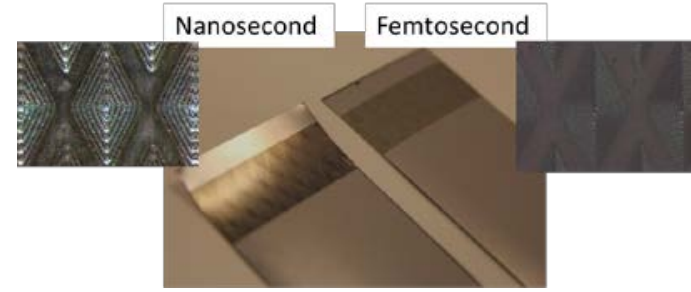

Fig. 6 Effects of pulse duration on metal sheet.

\subsection{Laser joining process}

In this present work, the joining process has been performed by passing through the polymer part which is sufficiently transparent to the laser wavelength. This joining methodology is named transmission joining (Fig. 7). The laser heats the metal plate. The heat is then transferred to the polymer by conduction. The polymer melts and fills the micro-structurations performed in the metal part. These last ones create a natural clamping system, keeping blocked the polymer inside the metal ensuring consequently the joining.

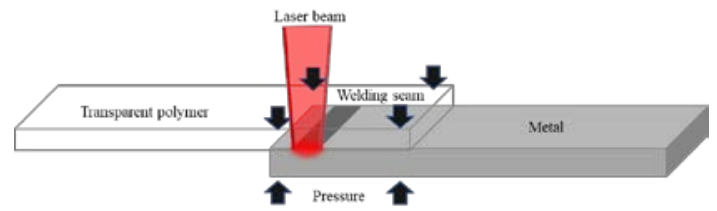

Fig. 7 Schematic picture of transmission joining.

A tensile test is performed thanks to a traction machine for determining the resistance of the joining and analyze, in this way, the influence of the global micro-structure density.

\section{Results and Discussion}

\subsection{Comparison of laser source and influence of GMD}

The study has been performed with three different laser sources, where the distinguishing parameter is the pulse duration. From nanosecond to femtosecond passing by the picosecond range, the study covers the short and ultrashort pulsed laser systems. The results for the hybrid combination between aluminum and polycarbonate $(2 \mathrm{~mm})$ plate are shown in Fig. 8. In this Figure, the structure density influences the tensile stress and depends on the laser source. The green point on the graph indicates the rupture of the polymer keeping intact the hybrid welding area.

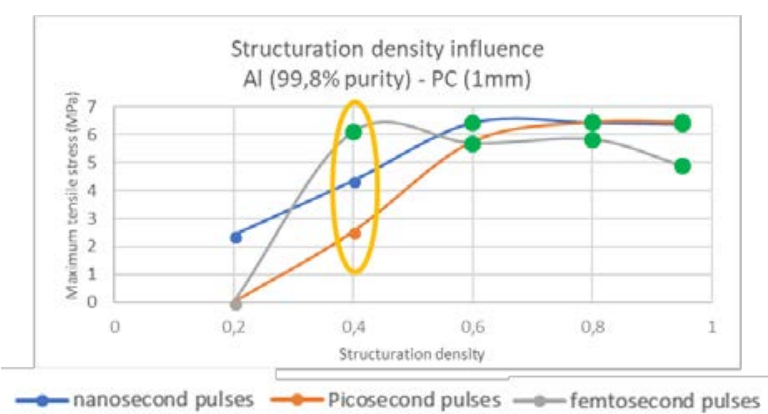

Fig. 8 Results of maximum tensile stress in function of GMD for nanosecond, picosecond and femtosecond laser. Green points indicate rupture of polymer keeping intact the joining area.

With femtosecond laser, the GMD can be reduced in comparison to the nanosecond and picosecond laser sources. In fact, for a GMD of 0.4, only the femtosecond laser can induce the rupture of the polymer without affecting the hybrid joining area. For nanosecond and picosecond lasers, a higher GMD is needed for ensuring the quality of the hybrid joining. Fig. 9(a) shows an example of the rupture of the polycarbonate after the tensile stress.
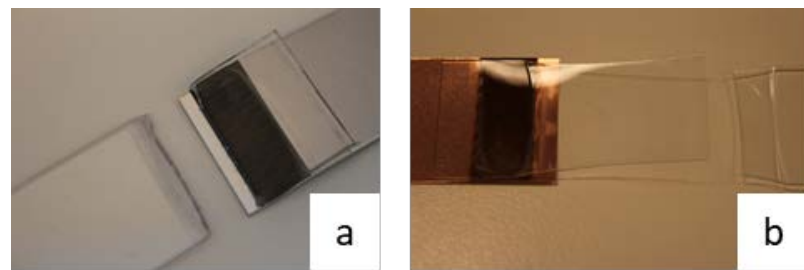

Fig. 9 Result of tensile test: Rupture of polymer keeping intact the joining area (a) $\mathrm{Al} / \mathrm{PC}$ and (b) $\mathrm{Cu} / \mathrm{PC}$.

A similar behavior is observed for the couples of materials copper/polycarbonate $(500 \mu \mathrm{m})$ and TiAl6V4/PLLA $(50 \mu \mathrm{m})$. For both hybrid joining, a structure density of 0.4 is only needed for reaching the rupture of the polymer keeping intact the joining when femtosecond laser is used.

Fig. 9(b) presents the joining after the tensile test for copper with polycarbonate.

The results also show that for very thin metal sheet like titanium alloy combined with biocompatible polymer PLLA, only the femtosecond laser can be used for performing the welding. In fact, the nanosecond laser induces the bending of the metal sheet making difficulties in the setting up of the welding. Furthermore, the picosecond laser structurations induce a degradation of the joining process and consequently a degradation of the PLLA.

\subsection{Influence of polymer thickness}

An important aspect of the study has been to determine how the thickness of the polymer influences the quality of the hybrid joining process, all other parameters remain constant. The study has been focused on the polycarbonate, and three different thicknesses have been compared $(0.5$ $\mathrm{mm}, 1 \mathrm{~mm}$ and $2 \mathrm{~mm}$ ).

Fig. 10 and 11 illustrate the results of the joining for structuration performed respectively with nanosecond laser and femtosecond laser.

For a structuration density of 0.6 , the rupture of the polymer can be achieved without affecting the joining area with nanosecond laser. However, if the polymer is of $2 \mathrm{~mm}$ thick, the nanosecond structurations cannot allow to reach the rupture of the polymer, the welding is only cohesive. Consequently, the nanosecond structuration induces a limitation in terms of polymer thickness.

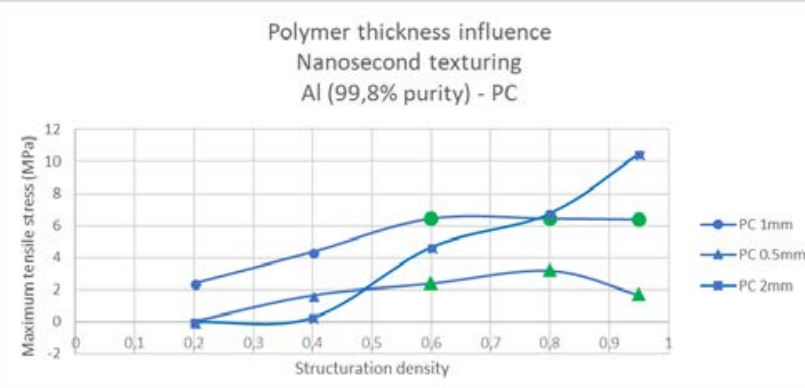

Fig. 10 Influence of polymer thickness on hybrid joining: nanosecond laser results. 
The femtosecond laser structurations allows to obtain good joining quality up to the rupture with a GMD of 0.6 for all tested thicknesses (Fig. 11). Due to this analysis, the thickness of the polymer could be increased in this case, allowing more flexibility in the material combination.

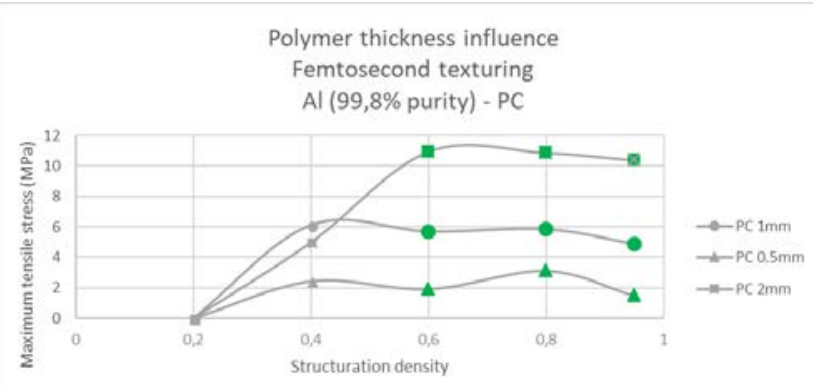

Fig. 11 Influence of polymer thickness on hybrid joining: femtosecond laser results.

These results confirm the importance of the selected laser source for the hybrid joining process depending of the quality of the joining expected. Moreover, it seems that the groove shape and the quality of the structuration process (no bending, less thermal affecting zones) help in the joining process of dissimilar materials.

\subsection{Influence of aspect ratio}

The aspect ratio is defined by the ratio between the groove depth ' $d$ ' and the groove width ' $W$ '. The analysis of this parameter will give a criteria for selecting the laser parameters in function of the geometrical parameters.

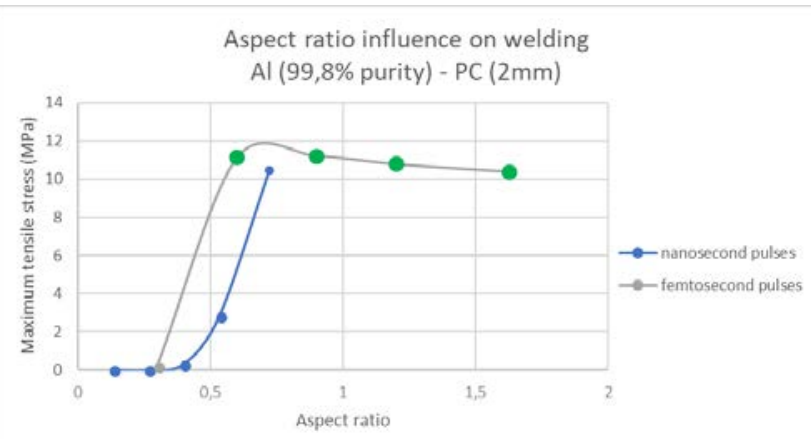

Fig. 12 Influence of aspect ratio on hybrid joining.

Fig. 1 shows the curves obtained for nanosecond and femtosecond lasers. For femtosecond laser, the joining will be the best when the depth of the groove will be higher than 0.6 times the groove width. In this case, it is possible to reach the rupture of the polymer. In the case of nanosecond laser, as it has shown in Fig. 10, the joining is cohesive. Nevertheless, if the criteria $\mathrm{d}>0.6 \mathrm{~W}$ is respected, the joining will be more resistant.

\subsection{Influence of welding surface}

$\mathrm{f}$ the surface treatment is decreased, it is possible to evaluate the maximal density to perform on the minimal surface for reaching the rupture of the polymer. Lasea has developed a methodology and a model in order to determine the minimal surface to micro-structure for achieving the expected resistance. The model is based on the determination of the rupture tensile stress of the material and the value of the tensile stress in function of the GMD.
Fig. 13 presents the result of the femtosecond process for the couple of dissimilar materials $\mathrm{Al} / \mathrm{PC}$. The optimum density is equal to 0.8 . It indicates the density for which the tensile stress is maximal.

Furthermore, the density of structuration must not be too high ( $>0.8)$ otherwise the tensile stress decreases. In fact, too many grooves in the metallic plate decreases the nontreated surface, the heat conduction is less efficient impacting directly the tensile stress of the joining

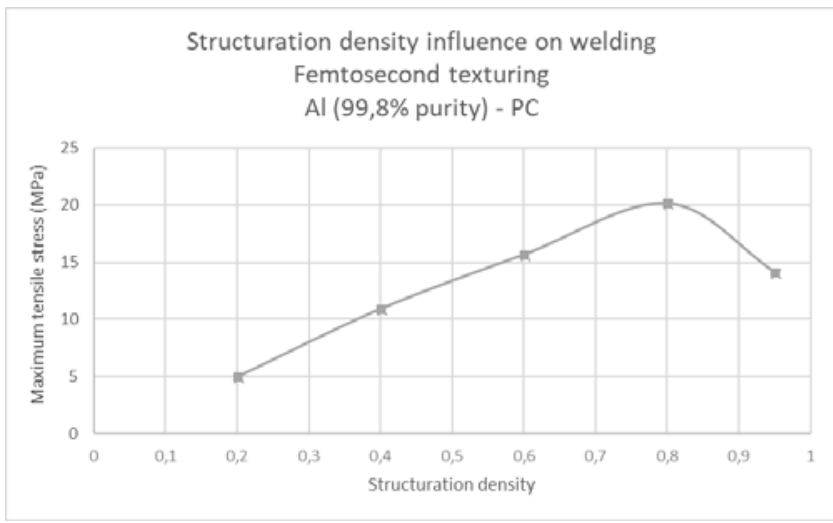

Fig. 13 Influence of aspect ratio on hybrid joining.

Thanks to the curve of the structure density, the minimal surface to treat can be determined. This curve is represented in Fig. 14.

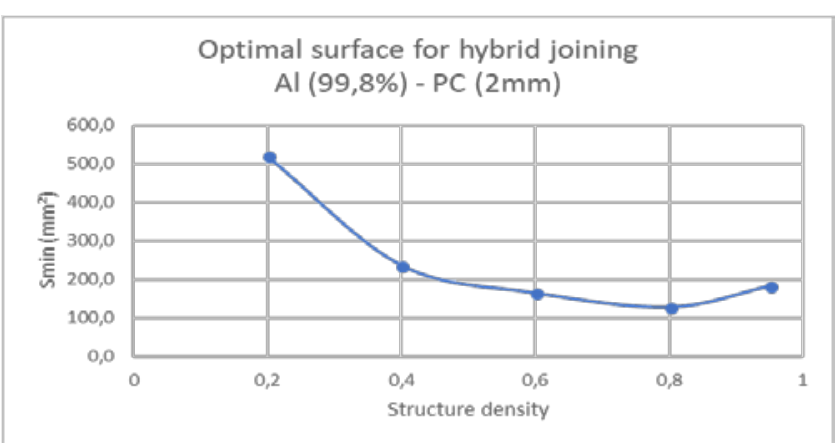

Fig. 14 Optimal surface for hybrid joining Al/PC.

A low structuration density ( 0.2 for example) needs higher surface for reaching the rupture of the polymer keeping intact the joining area. In fact, a minimal surface of structuration is necessary for clamping correctly the polymer inside the metal plate. This indication can allow to evaluate the resistance of the joining for a given surface or can give information of which surface to treat for an expected resistance.

\section{Conclusion}

Depending of the targeted application, the laser source should be adapted for reaching quality joining. Indeed, thermal effect and oxidation are, among others, undesirable effects which have to be avoided in some applications. In this work, a laser solution is proposed for joining metals with complex properties (reflectivity and thin thickness) and polymers while obtaining high joining quality and strength, i.e. up to the rupture of the polymer part without affecting the joining area. Based on micro-engraved structures with laser in the metallic part, this methodology is relevant for various metal/polymer couples and laser 
sources but could be also applied on other combinations of dissimilar material.

The present study has also developed a strategy and associated model for determining the minimal surface to treat or the structuration density for reaching the expected resistance.

\section{Acknowledgments and Appendixes}

We would like to acknowledge the assistance of the Wallonia Region for its financial support, in the frame of the project ID 9775.

\section{References}

[1] G. Verhaeghe, R. Beckschwarte and S. Parauchuri: ILS for manufacturing, 32, $\mathrm{n}^{\circ} 2$, (2017) 9.
[2] P. Kah, R. Suoranta, J. Martikainen and C. Magnus: Rev. Adv. Mater. Sci., 36, (2014) 152.

[3] J-P. Bergmann and M. Stambke: Phys. Proc., 39, (2012) 84.

[4] A. Roesner, S. Scheik, A. Olowinsky, A. Gillner, U. Reisgen and M. Schleser: Phys. Proc., 12, (2011) 370.

[5] P. Amend, S. Pfindel and M. Schmidt: Phys. Proc., 41, (2013) 98.

[6] A. Henrottin, JNPLI, (2016).

[7] A. Miam, G. Newaz, L. Vendra, N. Rahman, D.G. Georgiev, G. Auner, R. Witte and H. Herfurth: JMS: Material in medecine, 16, (2005) 229.

[8] N. Amanata, N.L. James and D.R. McKenziec: Medical Eng. \& Phys., 32, (2010) 690.

(Received: July 24, 2018, Accepted: November 18, 2018) 